\title{
Jamming and irreversibility
}

\author{
Julia Boschan $^{1} \cdot$ Stefan Luding ${ }^{1}$ Brian P. Tighe ${ }^{1}$ (I)
}

Received: 30 January 2019 / Published online: 21 June 2019

(c) The Author(s) 2019

\begin{abstract}
We investigate irreversibility in soft frictionless disk packings on approach to the unjamming transition. Using simulations of shear reversal tests, we study the relationship between plastic work and irreversible rearrangements of the contact network. Infinitesimal strains are reversible, while any finite strain generates plastic work and contact changes in a sufficiently large packing. The number of irreversible contact changes grows with strain, and the stress-strain curve displays a crossover from linear to increasingly nonlinear response when the fraction of irreversible contact changes approaches unity.
\end{abstract}

Keywords Jamming $\cdot$ Plasticity $\cdot$ Irreversibility

\section{Introduction}

Among many other topics in the physics of granular matter, Bob Behringer's research has had outsized impact on the field of jamming [1-3]. His measurements of the jump and subsequent power law growth in the contact number above a critical packing fraction [4] represents the first, and still one of the few [5-7], measurements of a major hallmark of isotropic jamming. His work on shear jamming [8], dilatancy [9], and contact force statistics [10, 11] upended the conventional view of the jamming phase diagram and illuminated how granular materials' rigidity encodes their loading history. Here, inspired by Bob's work, we ask how shearing can wipe out the memory of an initially isotropic state in a weakly jammed solid. In other words, how does irreversibility emerge near jamming?

Packings of soft spheres prepared at small but finite pressure are marginal solids-while their response to infinitesimal strains is elastic [1], a small shear stress suffices to instigate quasistatic plastic flow $[12,13]$. Recently there has been considerable interest in how the ensemble-averaged stress-strain curve for shear becomes nonlinear, and

This article is part of the Topical Collection: In Memoriam of Robert P. Behringer.

Brian P. Tighe

b.p.tighe@tudelft.nl

1 Process and Energy Laboratory, Delft University of Technology, Leeghwaterstraat 39, 2628 CB Delft, The Netherlands in particular on how the crossover from linear to nonlinear response depends on the distance to jamming [14-23]. The shear strain required to make or break a contact vanishes in the limit of large system sizes, so finite deformations necessarily involve topological changes to the contact network [24-28]. It is therefore natural to ask about the relationship between nonlinearity and plasticity, especially when one approaches (un)jamming. More precisely, we ask whether there is a correlation between the linear-to-nonlinear crossover and (ir)reversibile contact changes.

To probe nonlinearity and irreversibility near jamming, we study shear reversal in marginally jammed packings of athermal, frictionless, purely repulsive soft spheres. We begin from an isotropic state prepared at a targeted pressure $p$. We use this initial pressure (prior to shearing) to quantify the distance to unjamming at $p=0$. After preparation, the system is subjected to simple shear in small quasistatic steps to a maximum strain $\gamma_{m}$. The shearing direction is then reversed, and the system is returned to zero strain. A load is reversible if the stress follows the loading curve back to its initial value at zero strain. Reversible and irreversible deformations are illustrated in Fig. 1 with data from our simulations. This complements similar irreversibility under volumetric strain as observed in [21] and interpreted in terms of a history-dependent critical packing fraction.

The present work builds on results from Boschan et al. $[19,29]$, who studied the loading curve but did not consider shear reversal. The loading curve was found to be linear up to a strain scale $\gamma^{\dagger} \sim p$. After $\gamma^{\dagger}$ the stress continues to grow, albeit more slowly than an extrapolation of the initial linear 

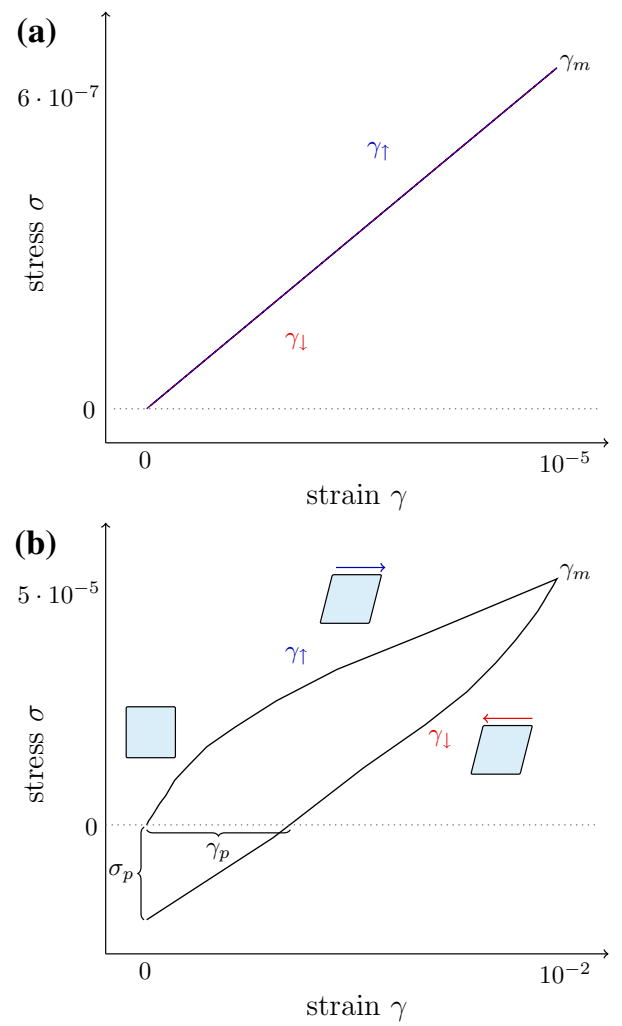

Fig. 1 Sample output from a loading-unloading cycle in simulations. a If deformation is reversible, the loading curve $\sigma\left(\gamma_{\uparrow}\right)$ and unloading curve $\sigma\left(\gamma_{\downarrow}\right)$ coincide. b In an irreversible deformation there is hysteresis, and the enclosed area is equal to the plastic work

trend. The crossover to steady plastic flow occurs later, at a distinct strain scale $\gamma_{y} \simeq 0.05$. Simulations of large amplitude oscillatory shear at finite rate also showed two distinct crossovers with identical scaling properties [22].

Boschan et al. [19] also studied contact changes, i.e. made and broken contacts during shearing. They found that the linear-to-nonlinear crossover at $\gamma^{\dagger}$ is also evident in the contact change statistics, as detailed in Sect. 4. It is plausible that contact changes are a proxy for irreversible rearrangements, but this must be verified-while rearrangements involve contact changes, not all rearrangements are irreversible [22, 30-35].

Here we probe nonlinearity and and irreversibility during a loading-unloading cycle. We first monitor the plastic work performed during the cycle, and then correlate these results to the statistics of contact changes at the particle scale. We find, first, that there is finite plastic work even when the ensemble-averaged stress-strain curve is linear. Consistent with this observation, we also find that irreversible contact changes accrue prior to the loss of linearity. Second, prior to $\gamma^{\dagger}$, some fraction of the contact changes are reversible. After $\gamma^{\dagger}$, when the stress-strain curve is nonlinear, essentially all contact changes are plastic.

\section{Model and methods}

We perform two-dimensional simulations of athermal frictionless disks, a standard model with a jamming transition [2]. Particles experience a spring-like force proportional to their overlap $\delta_{i j}=\left(R_{i}+R_{j}\right)-r_{i j}$, where $R_{i}$ and $R_{j}$ denote the radii and $r_{i j}$ is the length of the vector $\mathbf{r}_{i j}$ pointing from the center of particle $i$ to $j$. The contact force on particle $i$ due to particle $j$ is purely repulsive, and there is no interaction when the particles are not in contact,

$\mathbf{f}_{i j}^{\mathrm{el}}=\left\{\begin{array}{cc}-k\left(\delta_{i j}\right) \delta_{i j} \hat{\mathbf{r}}_{i j} & \delta_{i j} \geq 0 \\ \mathbf{0} & \delta_{i j}<0\end{array}\right.$

where a hat indicates a unit vector. We fix the units of stress by setting the spring constant $k$ and mean particle size to unity. The stress tensor is

$\sigma_{\alpha \beta}=-\frac{1}{2 V} \sum_{i j} \mathbf{f}_{i j, \alpha} \mathbf{r}_{i j, \beta}$,

where Greek indices denote Cartesian coordinates, and $V$ is the total area of the unit cell.

Initial conditions are created by randomly populating the bi-periodic simulation box and then using a nonlinear conjugate gradient energy minimization protocol to quench instantaneously to a local minimum of the elastic potential energy at fixed volume [36]. The box is then allowed to undergo small changes in size and shape to achieve a target pressure $p$ and zero shear stress-these are called "shear-stabilized" packings in the nomenclature of Dagois-Bohy et al. [37, 38]. Packings are bidisperse to avoid crystallization; we use the standard $[1,36] 50: 50$ mixture of small and large particles and a radius ratio of 1:1.4.

Once the initial state is prepared, we apply quasistatic simple shear using Lees-Edwards boundary conditions with small logarithmically-spaced steps ranging between $\Delta \gamma=10^{-8} \ldots 10^{-3}$. After each strain step the energy is reminimized [36] while holding the strain fixed, so particles follow quasistatic trajectories. Once a maximum strain $\gamma_{m}$ is reached, the direction of shear is reversed and the system is returned to zero strain, again via a series of small logarithmically-spaced steps.

In order to quantify irreversibility, we calculate the plastic work $W_{p}$ of the loading/unloading cycle,

$W_{p}=\oint \sigma \mathrm{d} \gamma=\int_{0}^{\gamma_{m}} \sigma_{\uparrow} \mathrm{d} \gamma_{\uparrow}-\int_{0}^{\gamma_{m}} \sigma_{\downarrow} \mathrm{d} \gamma_{\downarrow}$,

where upwards and downwards pointing arrows are used to indicate the loading and unloading curves, respectively. Clearly $W_{p}$ is zero when the response is reversible.

The phenomenology of a shear reversal test in weakly jammed soft spheres is illustrated in Fig. 1. In panel (a), the maximum shear strain $\gamma_{m}=10^{-5}$ is so small that no contact 
changes occur [26, 27]. The stress-strain curve is linear and the loading and unloading curves coincide. In panel (b), the maximum shear strain $\gamma_{m}=10^{-2}$ is substantially larger. On reversal the stress decreases but does not retrace the loading curve. The loading and unloading curves are both nonlinear. Because there is hysteresis, there is an associated plastic work. In addition to the plastic work, irreversibility can be quantified by the plastic strain $\gamma_{p}$ and a plastic stress $\sigma_{p}$, corresponding to the intercepts of the unloading curve with the $x$-and $y$-axis, respectively.

\section{Plastic work}

We perform shear reversal tests for a range of preparation pressures $p$ and varying maximum strain $\gamma_{m}$. Figure 2 illustrates loading and unloading curves for $p=10^{-4}$ and $\gamma_{m}$ ranging from $10^{-5}$ to $10^{-2}$ in half-decade steps. The result is representative of other pressures.

To quantify the appearance of irreversibility, we analyze the plastic work as a function of $\gamma_{m}$ and $p$, as shown in Fig. 3. We find nonzero $W_{p}$ for all investigated maximum

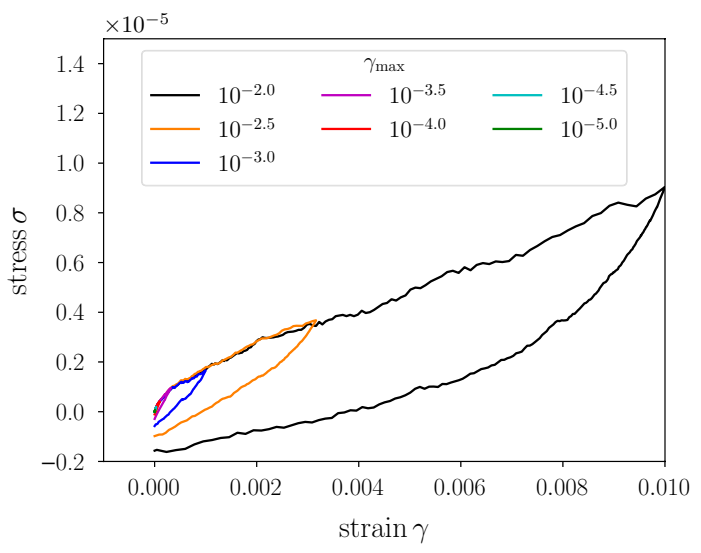

Fig. 2 Shear reversal tests for varying maximum strains $\gamma_{m}$ (see legend) at pressure $p=10^{-4}$ and system size $N=1024$ strains, which are as small as $10^{-5}$. (As noted above, packings of finite size can be sheared reversibly if the contact network remains unchanged, but this strain interval vanishes in the large system size limit [26, 27]). For each pressure $W_{p}$ has an approximately power law growth with $\gamma_{m}$, with an apparent exponent that varies with pressure.

To better understand the pressure dependence of $W_{p}$, we seek to collapse the data to a master curve. Anticipating a correlation with the onset of nonlinearity, we plot the rescaled variable $x \equiv \gamma_{m} / p \sim \gamma_{m} / \gamma^{\dagger}$. On the other axis we plot the rescaled work $\mathcal{W} \equiv W_{p} / p^{\rho}$ for some exponent $\varrho$. To motivate $\varrho$, we note that for small values of $\gamma_{m}$, the loading curve is associated with work $W_{\uparrow} \sim G_{0} \gamma_{m}^{2}$, where $G_{0} \sim p^{1 / 2}$ is the shear modulus for Hookean particles near jamming $[1,39,40]$. If we assume $G_{0}$ also sets the relevant scale for $W_{p}$ at small $\gamma_{m}$, then we expect $W_{p} \sim p^{1 / 2} \gamma_{m}^{2}$. Rearranging in favor of $\gamma_{m} / p$ gives $W_{p} / p^{5 / 2} \sim\left(\gamma_{m} / p\right)^{2}$, which requires $\varrho=5 / 2$. This prediction is tested in the $\log -\log$ plot of Fig. 3b, where we find data collapse to a curve with an initial slope of 2 . When $x \gg x^{*} \sim O(1)$ the plastic work grows more slowly with $\gamma_{m}$, with an exponent of roughly $3 / 2$,

$\mathcal{W} \sim\left\{\begin{array}{cc}x^{2} & x<x^{*} \\ x^{3 / 2} & x>x^{*}\end{array}\right.$

Plasticity is indeed sensitive to $\gamma^{\dagger}$, because data for $W_{p}$ collapse with the rescaled variable $x$. But irreversibility does not "turn on" when the ensemble-averaged stress-strain curve becomes nonlinear, as indicated by measurable $W_{p}$ even when the curve is linear.

\section{Contact changes}

We now seek to relate irreversibility to the evolution of contact changes during loading and unloading.

As first shown in Ref. [19] and verified below, the scale $\gamma^{\dagger}$ is apparent in the evolution of the number of made and
Fig. 3 a Plastic work versus maximum strain for varying pressures (see legend). b Collapse to a master curve with $\varrho=5 / 2$. The dashed lines have slopes 2 and $3 / 2$
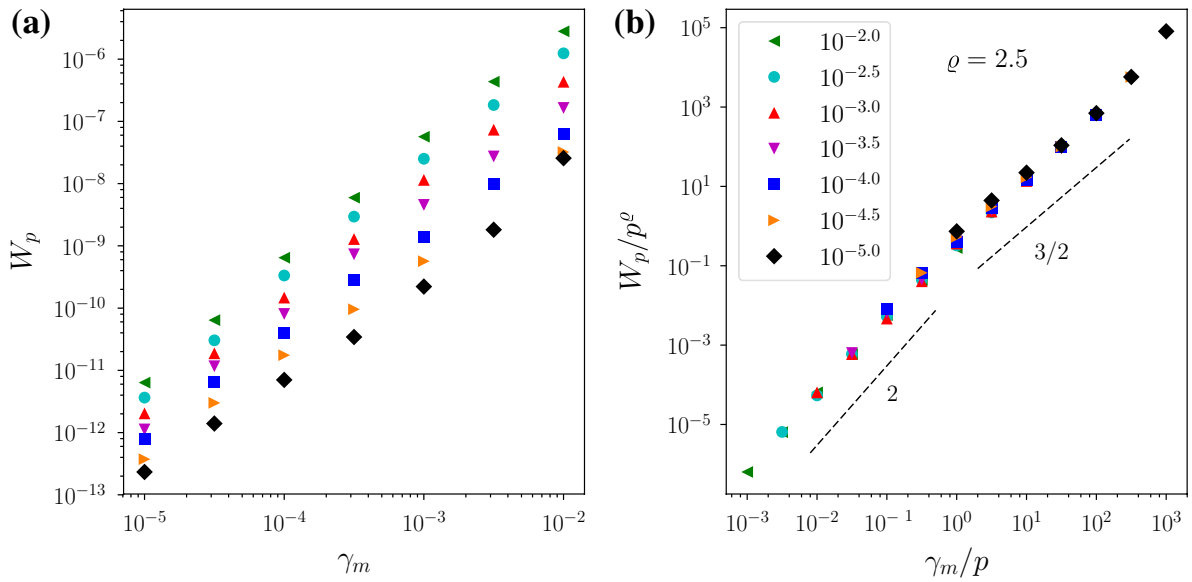
broken contacts per particle, which we refer to as the contact change density $n_{c c}(\gamma)$. We now monitor contact changes during unloading to see to what extent the original contact network is recovered (i.e. broken contacts are re-made and made contacts are re-broken). Contact changes are always identified with respect to the initial condition, even during unloading. The "plastic contact change density" $n_{c c}^{p}$, equal to $n_{c c}$ at the end of the unloading curve, is a measure of irreversible (i.e. plastic) contact changes.

Figure 4 depicts loading and unloading curves for three values of $\gamma_{m}$ and three different initial pressures $p=10^{-5}, 10^{-4}$ and $10^{-3}$. For the lowest $\gamma_{m}$, in panel (a), most contact changes are recovered at the end of the cycle and $n_{c c}$ has a nonzero slope. $n_{c c}^{p}$ is nevertheless nonzero, and it increases as $p$ tends to zero. Plastic contact changes also increase with increasing $\gamma_{m}$ (panels (b) and (c)). In the final panel a large fraction of the contact changes are unrecoverable, $n_{c c}$ hits the vertical axis with zero slope, and $n_{c c}^{p}$ is nearly equal to $n_{c c}\left(\gamma_{m}\right)$.

\subsection{Contact changes during loading}

Figure 5 depicts $n_{c c}$ during loading. The figure shows that data for different pressures can be collapsed to a master curve by plotting $\mathcal{N} \equiv n_{c c} /\left(\gamma^{\dagger}\right)^{1 / 2} \sim n_{c c} / p^{1 / 2}$ as a function of $y=\gamma / p \sim \gamma / \gamma^{\dagger}$. This collapse was first demonstrated in Ref. [19]; for completeness we present it in Fig. 5 using data from the present study. We find

$\mathcal{N} \sim\left\{\begin{array}{cc}y & y<y^{*} \\ y^{1 / 2} & y>y^{*}\end{array}\right.$

The crossover $y^{*} \sim O(1)$ is compatible with $x^{*}$ from the plastic work. For later reference, we note that

$n_{c c} \simeq a_{m} \gamma_{m}^{1 / 2}$

when $\gamma_{m}>\gamma^{\dagger}=y^{*} p$. We estimate $a_{m} \approx 3.7 \pm 0.1$ by fitting Eq. (6) to $\mathcal{N}$ for $y>10$.

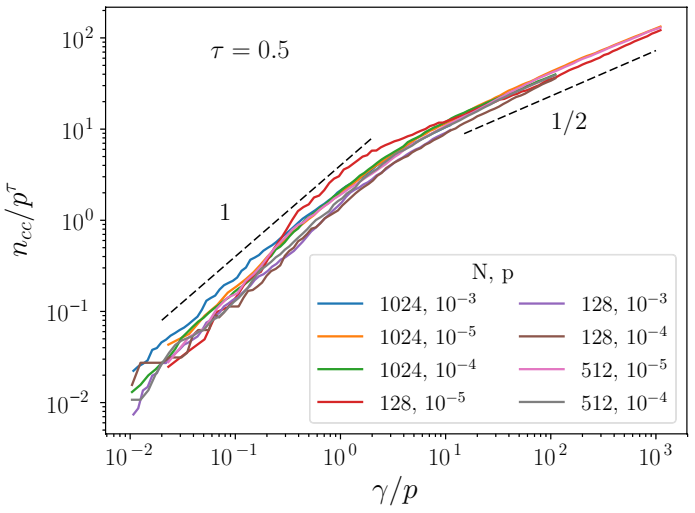

Fig. 5 When appropriately rescaled with the pressure, the contact change density $n_{c c}$ collapses to a master curve when plotted as a function of $\gamma / p$

We note that, by definition, $n_{c c}$ changes by an amount $1 / N$ when the system has undergone a strain $\gamma_{c c}$ sufficient to produce one contact change. Hence

$\frac{\mathrm{d} n_{c c}}{\mathrm{~d} \gamma} \approx \frac{1 / N}{\gamma_{c c}}$,

and the average strain interval between contact changes, $\gamma_{c c}$ can be read off from the slope of the curves in Fig. 4. (Alternatively, the probability of a contact change in the interval $[\gamma, \gamma+\mathrm{d} \gamma)$ is $\left.1 / \gamma_{c c}\right)$. In particular, when the loading curve is linear, there is a typical strain interval $\gamma_{c c} \sim p^{1 / 2} / N$ between contact changes. Van Deen et al. [26, 27] reached compatible results by directly resolving contact changes. As noted above, $\gamma_{c c}$ vanishes in the large system size limit.

\subsection{Contact changes after reversal}

To quantify to what extent the initial contact network can be recovered under reversal, we now monitor the plastic contact change density $n_{c c}^{p}$. Clearly $n_{c c}^{p}=0$ if the initial contact network is fully recovered. Figure 6 plots $n_{c c}^{p}$ as a function of $\gamma_{m}$ for three pressures and system sizes $N=128,512$, and 1024 .
Fig. 4 The contact change density $n_{c c}$ as a function of $\gamma / \gamma_{m}$ for $\mathbf{a} \gamma_{m}=10^{-5}, \mathbf{b} \gamma_{m}=10^{-4}$ and c) $\gamma_{m}=10^{-2}$ each at pressures $p=10^{-5}, 10^{-4}$ and $10^{-3}$ at $N=1024$. The solid lines indicate the loading the dashed lines the unloading curves

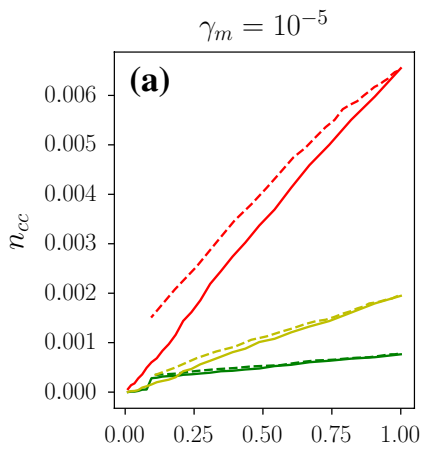

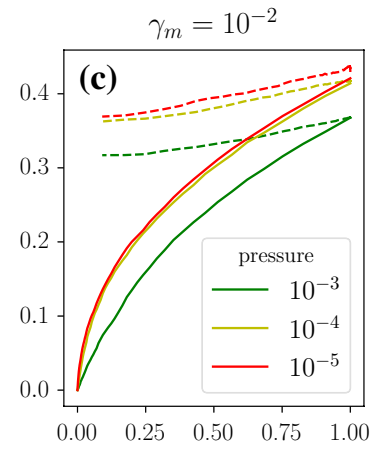


Fig. 6 a The plastic contact change density $n_{c c}^{p}$ as a function of maximum strain $\gamma_{m}$ at varying pressures $p$ and system sizes $N$. b Data collapse to a master curve. Dashed lines indicate the slopes 1 and $1 / 2$
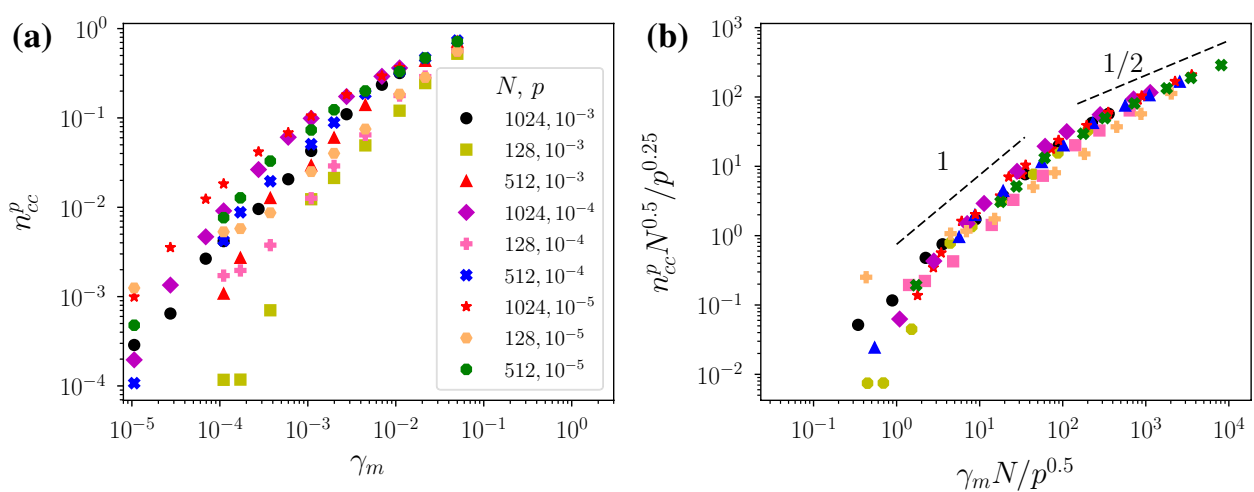

We find $n_{c c}^{p}$ is an increasing function of $\gamma_{m}$, and for a given $\gamma_{m}$ it is larger at smaller pressures. There is also dependence on $N$.

The system size-dependence in $n_{c c}^{p}$ suggests that the contact change strain $\gamma_{c c} \sim p^{1 / 2} / N$ plays a dominant role, as opposed to $\gamma^{\dagger} \sim p$. To test this hypothesis, we attempt to collapse data to a master curve by plotting as a function of $z \equiv \gamma_{m} N / p^{1 / 2} \sim \gamma_{m} / \gamma_{c c}$. We find collapse plotting $\mathcal{P} \equiv n_{c c}^{p} N^{1 / 2} / p^{1 / 4}$ versus $p^{1 / 2} / N$, as shown in Fig. $6 \mathrm{~b}$. The master curve is

$\mathcal{P} \sim\left\{\begin{array}{cc}z & z<z^{*} \\ z^{1 / 2} & z>z^{*}\end{array}\right.$

The crossover value $z^{*} \sim O\left(10^{2}\right)$. Therefore

$n_{c c}^{p} \simeq a_{p} \gamma_{m}^{1 / 2}$

after the system has undergone on the order of one hundred contact changes. The constant $a_{p} \approx 3.5 \pm 0.1$.

\subsection{Relating nonlinearity and irreversibility}

We can use the above observations to interpret the strain scale $\gamma^{\dagger}$ in terms of irreversibility. To this end, it is useful to introduce the "plastic fraction"

$f_{p}\left(\gamma_{m}\right)=\frac{\mathrm{d} n_{c c}^{p}}{\mathrm{~d} n_{c c}^{m}}$

where $n_{c c}^{m}$ is the value of $n_{c c}$ at the end of loading. $f_{p}$ quantifies the extent to which marginal contact changes tend to be plastic. If $f_{p}\left(\gamma_{m}\right)=0$, then all marginal contact changes in an infinitesimal interval around $\gamma_{m}$ are reversible. If $f_{p}=1$, all contact changes are plastic.

While a direct numerical evaluation of $f_{p}$ is noisy, we can infer its scaling properties by noting that

$f_{p}=\left(\frac{\mathrm{d} n_{c c}^{p}}{\mathrm{~d} \gamma_{m}}\right)\left(\frac{\mathrm{d} n_{c c}^{m}}{\mathrm{~d} \gamma_{m}}\right)^{-1}$.
From Eq. (9) it follows that

$\frac{\mathrm{d} n_{c c}^{p}}{\mathrm{~d} \gamma_{m}} \simeq \frac{a_{p}}{\gamma_{m}^{1 / 2}}$

in the $N \rightarrow \infty$ limit. Similarly, Eq. (6) implies that

$\frac{\mathrm{d} n_{c c}^{m}}{\mathrm{~d} \gamma_{m}} \simeq \frac{a_{m}}{\gamma_{m}^{1 / 2}}$

when $\gamma_{m}>\gamma^{\dagger}$. Thus $f_{p}$ plateaus at a value $a_{p} / a_{m} \approx 0.95$ when $\gamma_{m}>\gamma^{\dagger}$. In other words, after the linear-to-nonlinear crossover, around $95 \%$ of the subsequent contact changes are plastic. By contrast, for smaller values of $\gamma_{m}$ the plastic fraction evolves with strain.

\subsection{From contact changes to the stress-strain curve}

A remaining challenge is to determine how plastic events impact stress buildup. Here we make a first attempt. We expect irreversible contact changes to have an associated stress drop $\Delta \sigma_{p} / N$ due to an eigenvalue of the Hessian matrix going to zero $[41,42]$. Then we assume that the infinitesimal stress $\mathrm{d} \sigma$ generated by a strain $\mathrm{d} \gamma$ has both an elastic contribution and an offsetting stress release due to irreversible events

$\mathrm{d} \sigma=G_{0} \mathrm{~d} \gamma-\Delta \sigma_{p} \mathrm{~d} n_{c c}^{p}(\gamma)$.

Using Eq. (9) and rewriting in dimensionless form gives

$\frac{1}{G_{0}} \frac{\mathrm{d} \sigma}{\mathrm{d} \gamma}=1-\frac{\Delta \sigma_{p}}{p^{1 / 2} G_{0}}\left(\frac{p}{\gamma}\right)^{1 / 2}$.

It remains to determine the typical stress drop amplitude, $\Delta \sigma_{p}$. The scaling relation $\Delta \sigma_{p} \sim p$ suggests itself purely on dimensional grounds. Assuming this form then predicts that the right hand side of Eq. (15) depends on $\gamma$ and $p$ only via their ratio $\gamma / p$. Reassuringly, this is consistent with the linear-to-nonlinear crossover at $\gamma^{\dagger} \sim p$, and with recent measurements of the secant modulus during shear startup 
[19] and the storage modulus in oscillatory shear [17, 22]. We conclude that the typical stress drop is indeed linear in $p$. Eq. (15) can then be integrated to find

$\sigma \sim \begin{cases}p^{1 / 2} \gamma & \gamma<\gamma^{\dagger} \\ p \gamma^{1 / 2} & \gamma>\gamma^{\dagger}\end{cases}$

This stress-strain curve is compatible with $W_{p}$ in Fig. 3b, including the $\gamma^{3 / 2}$ scaling beyond $\gamma \approx \gamma^{\dagger}$.

The approach presented above is semi-empirical. A more fundamental motivation would require directly identifying plastic events to determine their frequency and associated stress drops. The necessary theoretical tools were recently developed in Refs. [42-44].

\section{Discussion}

We have investigated irreversibility at the macro and micro scale in systems near jamming, evidencing irreversibility in both the plastic work and the contact change statistics for small shear strains. Initially the average loading curve is linear and most contact changes are reversible. Increasing the maximum strain increases the number and fraction of plastic contact changes. For $\gamma>\gamma^{\dagger}$, the loading curve becomes nonlinear and nearly all contact changes are plastic. The onset of nonlinearity therefore corresponds not to the onset of irreversibility (as commonly assumed in continuum elasto-plastic theories), but to "fully developed" irreversibility, as reflected in the saturation of the plastic fraction $f_{p}$. This crossover occurs earlier for smaller $\gamma^{\dagger} \sim p$.

With hindsight, the above scenario is apparent in the contact change statistics. For small $\gamma_{m}$, as in Fig. 4a, the plastic contact change density is much smaller than $n_{c c}^{m}$, and the unloading branch of the $n_{c c}$ curve ends with a nonzero slope-indicating that shearing the system "a little bit further" to $\gamma_{\downarrow}<0$ would bring the system closer to its initial contact topology, i.e. fewer net contact changes. In contrast, for large $\gamma_{m}$, as in Fig. $4 \mathrm{c}, n_{c c}^{p}$ is nearly equal to $n_{c c}^{m}$, and the unloading curve is flat- the system has effectively lost all memory of its initial condition.

Our work has correlated the onset of nonlinearity at the macro scale to a particle scale crossover from reversible to irreversible contact changes. Both of these crossovers are sensitive to the proximity to jamming. We have also suggested a phenomenological approach to relate irreversible rearrangements to the form of the loading curve, highlighting the need for a deeper understanding of the statistics of stress drops during loading.

Acknowledgements We acknowledge financial support from the Dutch Organization for Scientific Research (NWO) and inspiring discussions with Bob Behringer.

\section{Compliance with ethical standards}

Conflict of interest The authors declare that they have no conflicts of interest.

Open Access This article is distributed under the terms of the Creative Commons Attribution 4.0 International License (http://creativeco mmons.org/licenses/by/4.0/), which permits unrestricted use, distribution, and reproduction in any medium, provided you give appropriate credit to the original author(s) and the source, provide a link to the Creative Commons license, and indicate if changes were made.

\section{References}

1. O'Hern, C.S., Silbert, L.E., Liu, A.J., Nagel, S.R.: Jamming at zero temperature and zero applied stress: the epitome of disorder. Phys. Rev. E 68, 011306 (2003)

2. van Hecke, M.: Jamming of soft particles: geometry, mechanics, scaling and isostaticity. J. Phys. Cond. Matt. 22, 033101 (2010)

3. Katgert, G., Tighe, B.P., van Hecke, M.: The jamming perspective on wet foams. Soft Matter 9(41), 9739-9746 (2013)

4. Majmudar, T.S., Sperl, M., Luding, S., Behringer, R.P.: Jamming transition in granular systems. Phys. Rev. Lett. 98, 058001 (2007)

5. Katgert, G., van Hecke, M.: Jamming and geometry of two-dimensional foams. EPL 92(3), 34002 (2010)

6. Desmond, K.W., Young, P.J., Chen, D., Weeks, E.R.: Experimental study of forces between quasi-two-dimensional emulsion droplets near jamming. Soft Matter 9, 3424-3436 (2013)

7. Jorjadze, I., Pontani, L.-L., Brujic, J.: Microscopic approach to the nonlinear elasticity of compressed emulsions. Phys. Rev. Lett. 110, 048302 (2013)

8. Bi, D., Zhang, J., Chakraborty, B., Behringer, R.P.: Jamming by shear. Nature 480, 355-358 (2011)

9. Ren, J., Dijksman, J.A., Behringer, R.P.: Reynolds pressure and relaxation in a sheared granular system. Phys. Rev. Lett. 110, 018302 (2013)

10. Majmudar, T.S., Behringer, R.P.: Contact force measurements and stress-induced anisotropy in granular materials. Nature $\mathbf{4 3 5}$, 1079-1082 (2005)

11. Zhang, J., Majmudar, T.S., Sperl, M., Behringer, R.P.: Jamming for a 2D granular material. Soft Matter 6, 2982 (2010)

12. Heussinger, C., Barrat, J.-L.: Jamming transition as probed by quasistatic shear flow. Phys. Rev. Lett. 102(21), 218303 (2009)

13. Tighe, B.P., Woldhuis, E., Remmers, J.J.C., van Saarloos, W., van Hecke, M.: Model for the scaling of stresses and fluctuations in flows near jamming. Phys. Rev. Lett. 105(8), 088303 (2010)

14. Knowlton, E.D., Pine, D.J., Cipelletti, L.: A microscopic view of the yielding transition in concentrated emulsions. Soft Matter 10, 6931-6940 (2014)

15. Coulais, C., Seguin, A., Dauchot, O.: Shear modulus and dilatancy softening in granular packings above jamming. Phys. Rev. Lett. 113, 198001 (2014)

16. Tighe, B.P.: Shear dilatancy in marginal solids. Granul. Matter 16(2), 203-208 (2014)

17. Otsuki, M., Hayakawa, H.: Avalanche contribution to shear modulus of granular materials. Phys. Rev. E 90, 042202 (2014)

18. Nakayama, D., Yoshino, H., Zamponi, F.: Protocol-dependent shear modulus of amorphous solids. J. Stat. Mech.: Theory Exp. 2016(10), 104001 (2016)

19. Boschan, J., Vågberg, D., Somfai, E., Tighe, B.P.: Beyond linear elasticity: jammed solids at finite shear strain and rate. Soft Matter 12, 5450-5460 (2016) 
20. Goodrich, C.P., Liu, A.J., Sethna, J.P.: Scaling ansatz for the jamming transition. PNAS 113(35), 9745-9750 (2016)

21. Kumar, N., Luding, S.: Memory of jamming-multiscale models for soft and granular matter. Granul. Matter 18(3), 58 (2016)

22. Dagois-Bohy, S., Somfai, E., Tighe, B.P., van Hecke, M.: Softening and yielding of soft glassy materials. Soft Matter 13, 90369045 (2017)

23. Baumgarten, K., Tighe, B.P.: Normal stresses, contraction, and stiffening in sheared elastic networks. Phys. Rev. Lett. 120(14), 148004 (2018)

24. Combe, G., Roux, J.-N.: Strain versus stress in a model granular material: a Devil's staircase. Phys. Rev. Lett. 85(17), 3628 (2000)

25. Schreck, C.F., Bertrand, T., O'Hern, C.S., Shattuck, M.D.: Repulsive contact interactions make jammed particulate systems inherently nonharmonic. Phys. Rev. Lett. 107(7), 078301 (2011)

26. van Deen, M.S., Simon, J., Zeravcic, Z., Dagois-Bohy, S., Tighe, B.P., van Hecke, M.: Contact changes near jamming. Phys. Rev. E 90, 020202 (2014)

27. van Deen, M.S., Tighe, B.P., van Hecke, M.: Contact changes of sheared systems: scaling, correlations, and mechanisms. Phys. Rev. E 94, 062905 (2016)

28. Kumar, N., Luding, S., Magnanimo, V.: Macroscopic model with anisotropy based on micro-macro information. Acta Mech. 225(8), 2319-2343 (2014)

29. Boschan, J., Vasudevan, S.A., Boukany, P.E., Somfai, E., Tighe, B.P.: Stress relaxation in viscous soft spheres. Soft Matter 13(38), 6870-6876 (2017)

30. Lundberg, M., Krishan, K., Xu, N., O'Hern, C.S., Dennin, M.: Reversible plastic events in amorphous materials. Phys. Rev. E 77, 041505 (2008)

31. Regev, I., Lookman, R., Reichhardt, C.: Onset of irreversibility and chaos in amorphous solids under periodic shear. Phys. Rev. E 88, 062401 (2013)

32. Saitoh, K., Magnanimo, V., Luding, S.: A master equation for the probability distribution functions of forces in soft particle packings. Soft Matter 11, 1253-1258 (2015)
33. Regev, I., Weber, J., Reichhardt, C., Dahmen, K.A., Lookman, T.: Reversibility and criticality in amorphous solids. Nat. Commun. 6, 8805 (2015)

34. Priezjev, N.V.: Reversible plastic events during oscillatory deformation of amorphous solids. Phys. Rev. E 93(1), 013001 (2016)

35. Saitoh, K., Oyama, N., Ogushi, F., Luding, S.: Transition rates for slip-avalanches in soft athermal disks under quasi-static simple shear deformations. Soft Matter (2019)

36. Koeze, D.J., Vågberg, D., Tjoa, B.B.T., Tighe, B.P.: Mapping the jamming transition of bidisperse mixtures. EPL 113(5), 54001 (2016)

37. Dagois-Bohy, S., Tighe, B.P., Simon, J., Henkes, S., van Hecke, M.: Soft-sphere packings at finite pressure but unstable to shear. Phys. Rev. Lett. 109, 095703 (2012)

38. Goodrich, C.P., Dagois-Bohy, S., Tighe, B.P., van Hecke, M., Liu, A.J., Nagel, S.R.: Jamming in finite systems: stability, anisotropy, fluctuations, and scaling. Phys. Rev. E $90(2), 022138$ (2014)

39. Wyart, M.: On the rigidity of amorphous solids. Ann. de Phys. 30, 1 (2005)

40. Tighe, B.P.: Relaxations and rheology near jamming. Phys. Rev. Lett. 107, 158303 (2011)

41. Maloney, C.E., Lemaître, A.: Amorphous systems in athermal, quasistatic shear. Phys. Rev. E 74(1), 016118 (2006)

42. van Deen, M.S.: Mechanical response of foams: elasticity, plasticity, and rearrangements. Ph.D. thesis (2016)

43. Gartner, L., Lerner, E.: Nonlinear plastic modes in disordered solids. Phys. Rev. E 93, 011001 (2016)

44. Lerner, E.: Micromechanics of nonlinear plastic modes. Phys. Rev. E 93(5), 053004 (2016)

Publisher's Note Springer Nature remains neutral with regard to jurisdictional claims in published maps and institutional affiliations. 